\title{
THE NEYMAN-PEARSON THEORY AFTER FIFTY YEARS
}

E. L. LEHMANN University of California, Berkeley

ABSTRACT: To commemorate the 50th anniversary of the Neyman-Pearson paradigm, this paper sketches some aspects of its development during the past half-century. In particular, the relevance of this approach to data analysis and Bayesian statistics is discussed.

\section{INTRODUCTION}

The Neyman-Pearson theory of hypothesis testing was developed by its two authors during the years 1926-1934. ' However, its official birthday may be assigned to 1933, which saw the publication of the seminal paper "On the Problem of the Most Efficient Tests of Statistical Hypotheses." In this paper, the authors provided both the first complete statement of their new approach and some of its most important applications.

Research partially supported by National Science Foundation Grant MCS82-01498.

From Proceedings of the Berkeley Conference in Honor of Jerzy Neyman and Jack Kiefer, Volume I, Lucien M. Le Cam and Richard A. Olshen, eds., copyright (C) 1985 by Wadsworth, Inc. All rights reserved.

${ }^{1}$ Accounts of the collaboration can be found in Pearson's recollections (1966) and in the Neyman biography by Reid (1983). 
The theory contains three principal ingredients:

(i) a parometric model specifying the assumed family of distributions of the observable random variables;

(ii) a criterion measuring the performance of a given statistical procedure, namely, the power function of the test in question;

(iii) the advocacy of optimality: that the most desirable test is obtained by maximizing the power.

Despite the fact that these ideas had their forerunners in the work of Fisher, ${ }^{2}$ the Neyman-Pearson paradigm formulated for the first time a clear program and provided a completely novel approach to hypothesis testing, the first "exact" small-sample theory of its kind.

It is the purpose of this paper to commemorate the 50th anniversary of the NP theory by considering some aspects of its development during the past half-century and to inquire into its relevance for statistics today. A particular concern will be the contribution the NP approach can make to data analysis and Bayesian statistics. All three of these paradigms have had extensive developments, generalizations, and elaborations during the period in question.

Data analysis had its origin in descriptive statistics, which dealt with the description and summary of data without making any assumptions, in particular, without assuming an underlying stochastic model. Under the leadership of Tukey (see, for example, his paper of 1962), many new techniques were developed and new tasks added such as the discovery of underlying structure and of transformations to simplify such structure. Recent treatments of the subject can be found in the books by Tukey (1977), Mostelier and Tukey (1977), and Hoaglin, Mosteller, and Tukey (1983). For critiques, see, for example, Dempster (1983) and Mallows (1983).

The Neyman-Pearson theory, which was orfginally concerned only with hypothesis testing, was adapted by Neyman (1937) to deal also

\footnotetext{
${ }^{2}$ Particularly in his fundamental paper of 1922, which dealt with point estimation but also emphasized parametric models and the fact that maximum likelihood estimators minimize the asymptotic variance. Fisher was preceded by Edgeworth.
} 
with estimation by confidence sets and was generalized by Wald to his general decision theory, in which the power function was replaced by a general risk function, new optimality criteria were introduced, and the restriction to parametric models was jettisoned (although in Wald's final exposition of 1950 the examples were all parametric).

Bayesian statistics had come to a first flowering in the work of Laplace. It had been pushed aside in deliberate efforts by Fisher, and Neyman and Pearson, to bulld an "objective" theory and was reawakened by L. J. Savage ${ }^{3}$ (particularly in his 1954 book) and earlier, from a somewhat different point of view, by Jeffreys (1939). One of its principal features is the assumption that the parameters of the parametric model should themselves be viewed as random variables whose distribution is assumed to be known.

The needs that lie behind the development of data analysis and Bayesian statistics had their impact also on the NP theory. In particular, the desire to free statistical analysis from the stringent and unreliable assumptions of parametric models moved the NP theory in the direction of data analysis, and the need to incorporate prior information and subjective impressions brought it closer to Bayesian statistics. The two trends will be sketched in Sections 2-4.

\section{ROBUSTNESS AND ADAPTATION}

A typical example of the classical NP approach is the normal two-sample problem. If one assumes that $X_{1}, \ldots, X_{m}$ and $Y_{1}, \ldots, Y_{n}$ are independent with normal distributions

$$
\begin{aligned}
& X_{1}, \ldots, X_{m}: N\left(\xi, \sigma^{2}\right) \\
& Y_{1}, \ldots, Y_{n}: N\left(\eta, \sigma^{2}\right),
\end{aligned}
$$

then the optimal test of the hypothesis $n=\xi$ against the one-sided alternatives $\eta>\xi$ under a variety of optimality criteria (UMP

\footnotetext{
${ }^{3}$ Savage acknowledged the profound influence on his thinking of de Finetti, as presented, for example, in de Finetti (1937).
} 
unbiased, UMP invariant, most stringent, and so forth) is the twosample t-test.

However, the validity of this model is frequently doubtful: the distribution of the errors may not be normal, the two variances not be equal, the observations not be independent. We shall here consider only the effect of non-normality, and hence the model

$$
\begin{aligned}
& X_{1}, \ldots, X_{m}: F(x-\xi) \\
& Y_{1}, \ldots, Y_{n}: F(y-\eta),
\end{aligned}
$$

where $F$ is unknown. Before embarking on this program, we should mention a type of model that is intermediate between (2.1) and (2.2). In this formulation, it is assumed that the distribution $F$ in (2.2) is approximately normal, for example, that

$$
E(x)=(1-\varepsilon) \Phi(x)+\varepsilon H(x)
$$

where $\varepsilon$ is given and $H$ is an arbitrary distribution function. Such models, which have been studied by Huber [see, for example, Huber (1981)], will not be considered in the present paper.

The first question that was asked about the robustness of the $t$-test concerned the actual level $\alpha_{m, n}(F)$ of the $t$-test carried out at nominal level $\alpha$ when the model is given by (2.2). This question was raised quite early; for a review of some of the relevant literature, see Tan (1982). It immediately follows from the central limit theorem and slutsky's theorem that

$$
\alpha_{m, n}(E) \rightarrow \alpha \text { as } m, n \rightarrow \infty
$$

for any $E$ with finite second moment. The same type of argument shows further that, for large $m$ and $n$, not only the level but also the power of the t-test is approximately equal to the power predicted under the normal model.

If the value of the significance level is taken very seriously, the approximate nature of the level under (2.2) may be considered inadequate. The difficulty can be overcome by a modification of the t-test, the permutation (or randomization) $t$ test due to $\mathrm{R}$. A. Fisher (1935). In this test the t-statistic is compared not with all the values it takes on as the $X^{\prime} s$ and $Y^{\prime} s$ range over the sample space but 
only with the values obtained by permuting the $m+n$ observed values $\left(x_{1}, \ldots, x_{m}, y_{1}, \ldots, y_{n}\right)$. The resulting test has a level that is independent of $F$ in the modei (2.2). In addition, it has asymptotically the same power as the t-test (Hoeffding, 1952). It thus appears to be an ideal solution of the robustness problem.

Unfortunately, this conclusion overlooks the fact that, if the normal model is wrong, the t-test loses its optimality properties and may actually not be a particularly desirable test to use. The distinction is that between robustness of validity (or, more generally, performance robustness) and robustness of efficiency, introduced by Tukey and McLaughlin (1963). It is closely related to the concepts of criterion and inference robustness proposed by Box and Tiao (1964). The t-test and its permutation version are performance-robust in that their performance is approximately that asserted by the normal theory even under model (2.2). However, since the efficiency of these tests does not continue to hold even approximately in (2.2) when $F$ is not normal, performance robustness is not as strong a recommendation as appears at first sight.

There exist, in fact, tests (based on the rank of the observations) whose significance level is independent of $F$ and whose power for many realistic distributions $F$ is much higher than that of the t-test. A particularly striking example is the normal-scores test $N$, whose asymptotic relative efficiency (ARE) $e_{N, t}\left(F^{\prime}\right)$ with respect to the t-test satisfles (Chernoff and Savage, 1958)

$$
e_{N, t}(F) \geqslant 1 \text { for all } E \text {, }
$$

with equality holding only when $F$ is normal, and with $e_{N, t}(F)$ becoming arbitrarily large when the tail of $F$ is sufficiently heavy. The test $N$ has the asymptotic optimality property of maximizing inf $e_{N, t}(F)$ in model (2.2).

In this solution to the robustness problem the original model (2.1) still plays a central (though implicit) role because it sets up the t-test as the standard with which other tests are being compared. If we want to treat (2.2) without any special relevance to the normal case, a more natural efficiency measure of a test is its absolute 
Lehmann

efficiency $e(E)$, that 1 , its efficiency relative to the best parametric test of $H: \Delta=\eta-\xi=0$ when $F$ is known (for example, the UMP invariant test). Stein (1956) suggested the existence of a test that would satisfy

$$
e(E)=1
$$

for all sufficiently smooth $F$. The program of working out such adaptive procedures (which adapt themselves to the unknown true $F$ ) has been carried out for a number of problems. For the estimation of the center of a symmetric distribution on the basis of a sample $X_{1}, \ldots$, $X_{n}$, for example, fully efficient adaptive estimators were obtained by Beran (1974) and Stone (1975). The latter shows that the smal1-sample efficiency of his estimator is of the order of .9 for a variety of distributions of quite different shapes (such as normal, Cauchy, and double-exponential) for sample sizes as small as $n=40$. The conditions under which adaptation is possible have been investigated by Bicke1 (1982).

\section{RELATION WITH DATA ANALYSIS}

For removing the assumption of normality from model (2.1), adaptive tests and estimators provide the ultimate answer. However, they seem to be far from the methods and concerns of data analysis as an approach to statistics without any modeling. In fact, they are closer than appears at first sight.

Adaptive procedures begin by estimating the unknown distribution $F$ or its density $f$; they then apply the procedure that would be best if the estimated $f$ were the true one. However, the problem of estimating an unknown density $f$ is quite close to that of describing a data set by means, say, of a histogram. Exactly the same issues arise in both (interval width, smoothing, etc.); see, for example, Scott (1979), Freedman and Diaconis (1981), and Hoaglin, Mosteller, and Tukey (1983). 
There is, of course, a difference between the two methods of attack. The modeling approach can measure and compare the performance of different procedures; it can ask how close the estimated density is likely to be to the true one and obtain the interval width that provides the best convergence rate. However, this requires making some assumptions: for example, that the observations are $n$ independent realizations of a random variable whose distribution has a smooth density.

The Neyman-Pearson program as represented by (ii) and (iii) of the Introduction can be applied not only to data description but also to other aspects of data analysis. We shall briefly consider its contribution to two of those: data summaries and exploration of underlying structure.

To summarize a simple one-dimensional batch of data, one calculates statistics that indicate, for example, the center and the spread of the batch. If one is willing to assume that the observations $X_{1}, \ldots, X_{n}$ are a sample from a distribution $F$, one will be concerned instead with measures $\mu(F)$ of the center and spread of $F$ (or of skewness, Kurtosis, etc.), and the summary statistics become estimators of the unknown $\mu(E) .{ }^{4}$ Some of the concerns about such summarles can be shared by the two approaches; resistance to outliers is an important example. However, the assumption of an underlying probability model provides new possibilities. In particular, one can now again measure and compare the performance of different estimators. The choice of suitable measures $\mu(F)$ (and their estimators) from this point of view is discussed in Bickel and Lehmann (1975/1976).

Methods for discovering underlying structure (including the choice of transformations to simplify the structure) can again be strengthened by making some assumptions. As an example, we mention recent work concerned with model selection that assumes a class of possible models of varying complexity; see, for example, Stone (1981).

\footnotetext{
${ }^{4}$ Typically $\mu(F)$ is estimated by $\mu\left(F_{n}\right)$, the functional $\mu$ evaluated at the empirical distribution function $F_{n}$.
} 
The data are used to choose a suitable model within this class. An interesting new feature of this application of the Neyman-Pearson-Wald approach is that the loss function must include a component representing the complexity of the model.

It thus appears that many of the problems considered in data analysis can also be studied from the NPW point of view. The latter will always require some (although possibly rather weak) assumptions, and this can never quite match the freedom of a totally unrestrained examination of the data. On the other hand, it has the advantage of allowing evaluation and comparison of different methods. In addition, it may permit the extrapolation of findings to other data sets, whereas a purely data-analytic approach is bound to the data set under consideration.

\section{RELATION WITH BAYESIAN INFERENCE}

The move toward data analysis has as its aim a weakening of assumptions that are frequently over-detailed and unrealistic. In the other direction, the Bayesian approach tries to take account of additional information, not represented in the classical models, that affects our expectations regarding the parameter values likely to obtain. This is achieved by postulating that the parameters themselves are random variables (though unobservable) with a known probability distribution.

Non-Bayesians and most Bayesians appear to be in agreement on two basic facts:

(i) The procedures one wants to use are Bayes' procedures with respect to some prior distribution (or at least limits of such procedures). For Bayesians this follows from first principles. From a decision theoretic point of view it is a consequence of the fact that only Bayes' procedures and their limits are admissible.

(ii) Obtaining the appropriate prior distribution is usually a difficult task. Different Bayesian ideas of how to go about this are represented, for example, by the books of Raiffa and Schlaifer (1961), Box and Tiao (1964), and Lindley (1965). In the present section, we shall briefly discuss the contribution of decision theory to this problem. 
Since a large-sample approach often brings great simplification, let us begin by considering the asymptotic situation of a large number of i.i.d. random variables having density $f(x, \theta)$, where $\theta$ is real-valued and has prior density $\pi(\theta)$ with $\pi(\theta)>0$ for all $\theta$ and $E|\theta|<\infty$. Then, under suitable regularity conditions on $f(x, \theta)$, the Bayes' estimator $\delta_{\pi, n}$ of $\theta$ with respect to squared error loss satisfies $^{5}$

$$
\sqrt{n}\left(\delta_{\pi, n}-\theta\right) \rightarrow N(0,1 / I(\theta)) \text { in law, }
$$

where $I(\theta)$ is the amount of information each $X_{i}$ contains about $\theta$. See, for example, Lehmann (1983), Section 6.7.

Thus, to the accuracy of this approximation, the Bayes' procedures share the asymptotic optimality of maximum likelihood estimators: they are efficient. However, it is a consequence of this result that the theory cannot distinguish between them. In fact, the Bayes' estimators corresponding to two different prior densities $\pi$ and $\pi^{\prime}$ satisfy

$$
\sqrt{n}\left(\delta_{\pi, n}-\delta_{\pi^{\prime}, n}\right) \rightarrow 0 \text { in probability }
$$

Thus, large-sample theory does indeed greatly simplify the situation. Unfortunately it simplifies it too much.

A crucial assumption in these considerations is that $\pi(\theta)>0$ for all $\theta$, because a Bayes' procedure pays no attention to parameter values that are impossible a priori. This suggests that optimal risk can be attained most rapidly (in a minimax sense) if $\pi$ is not too uneven, i.e., if there are no $\theta$-values for which $\pi(\theta)$ is relatively very low. Actually, such an argument should also take into account the amount of information the sample contains about different values of $\theta$, which then points to an uninformative prior, and results in an approach that some authors call objective Bayesian. Furthermore, in problems in which invariance considerations are applicable, the decision theoretic invariance principle leads directly, even for small

\footnotetext{
${ }^{5}$ However, see Diaconis and Freedman (1983) and Freedman and Diaconis (1983).
} 
samples, to the Bayes' solution corresponding to an invariant (and hence noninformative) prior.

If, based on previous experience and perhaps other less tangible and more subjective impressions, one has a preferred prior distribution, decision theory suggests an alternative approach. Compare the behavior of procedures corresponding to a number of representative priors, including the prior of one's choice, and adopt the latter if its performance is satisfactory. Essentially the same strategy is recommended in a book, written from a Bayesian point of view, by J. Berger (1980). In the contrary case, one may want to tone down one's prior opintons in the direction of a noninformative prior.

A compromise of this $k$ ind is offered by the theory of restricted Bayes solutions. Suppose one has arrived at a preferred prior $\Lambda$, which leads to very high values of the risk function for some parameter values and about which one does not feel very confident. Consider for a moment the minimax solution as a competitor that has a much smaller maximum risk $M$ but which corresponds to a prior very different from $\Lambda$. As a compromise between these alternatives, let us limit the allowable maximum risk not to its minimum value $M$ but to $M(1+\varepsilon)$, so that the risk function is required to satisfy

$$
R(\theta, \delta) \leqslant M(1+\varepsilon) \text { for all } \theta \text {. }
$$

The restricted Bayes' procedure $\delta_{\Lambda, \varepsilon}$ is then obtained by minimizing the Bayes' risk $\int R(\theta, \delta) d \Lambda(\theta)$ subject to (4.3); see Hodges and Lehmann (1953). The choice of $\varepsilon$ in (4.3) will reflect both the confidence one places on $\Lambda$ and the cost of excessively high risk. Applications of this approach have been developed by Efron and Morris (1971), Bickel (1983), and Jelithovschi (1984).

\section{CONCLUSIONS AND ACKNOWLEDGMENTS}

The Neyman-Pearson theory makes two principal recommendations: Evaluate the behavior of statistical procedures in terms of some performance criterion, and then opt for the procedure that performs best. The 
application of these recommendations requires a probabilistic model for the observations, although such models can be much broader than those originally envisaged by Neyman and Pearson.

As has been indicated in the preceding sections, the first of these recommendations can be usefully applied to a wide spectrum of statistical techniques ranging from procedures inspired by data analysis to those obtained from Bayestan principles. It is difficult to imagine statistics as a subject in which this idea would not play a central role.

Complete reliance on optimality, on the other hand, suffers from some drawbacks. In particular,

(i) procedures that minimize the risk may be quite unsatisfactory in other respects such as robustness or interpretability; most optimality criteria lead to solutions only in fairly simple problems.

As a consequence, an optimal solution (if it exists) should not be considered the ultimate answer, but its performance should be studied, just as that of any other proposed procedure, from all relevant points of view. On the other hand, a procedure that is best in some way is ut value ds a pencnmark wath which rnmnottno nronodwroc nan ho nompared. It shows how well one can do in this particular direction and how much is lost when other criteria have to be taken into account.

It is suggested in Sections 3 and 4 that the procedures obtained from a decision theoretic point of view may not differ as much from those reached via a data analytic or Bayestan philosophy as is frequently claimed and that all three ways of treating problems can have contributions to make. This point has been made by other authors.

The relationship between decision theory and data analysis is explored by Thisted (1981) and in the book by Hoaglin, Mosteller, and Tukey (1983). Regarding the uses of decision theory in a Bayesian setting, the work of Berger (1980) has already been mentioned. A different combination of Bayesian and Neyman-Pearson theory has been recommended by Box (1980), who proposes a Bayesian approach to the 
Lehmann

estimation of the parameters of the model and a test based on sampling considerations for testing the model.

Finally, it was shown how all three lines of attack can be used in a substantive study by Mosteller and Wallace (1964), and a plea for "ecumenism" in statistics is put forth by Box (1983).

\section{REFERENCES}

Andrews, D. F. (1978). Article on "Data Analysis, Exploratory." In Internat. Encycl. of Statist. Kruskal and Tanur, eds. Free Press, New York.

Beran, R. (1974). Asymptotically efficient adaptive rank estimates in location models. Ann. Statist. 2, 63-74.

Berger, J. (1980). Statistical Decision Theory. Springer, New York.

Bicke1, P. J. (1981). Minimax estimation of the mean of a normal distribution when the parameter space is restricted. Ann. Statist. 9, 1301-1309.

Bicke1, P. J. (1982). On adaptive estimation. Ann. Statist. 10, $647-671$.

Bicke1, P. J. (1984). Parametric robustness. Ann. Statist. 12.

Bicke1, P. J., and Lehmann, E. L. (1975/1976). Descriptive statistics for nonparametric models. Ann. Statist. 3, 1038-1069; $4,1139-1158$.

Box, G. E. P. (1980). Sampling and Bayes' inference in scientific modelling and robustness. J. Roy. Statist. Soc. (A) 143, 383-430.

Box, G. E. P. (1983). An apology for ecumenism in statistics. In Scientific Inference, Data Analysis, and Robustness. Box, Leonard, and Wu, eds. John Wiley, New York.

Box, G. E. P., and Tiao, G. C. (1964). A note on criterion robustness and inference robustness. Biometrika 51, 169-173.

Box, G. E. P., and Tiao, G. C. (1973). Bayesian Inference in Statistical Analysis. Addison-Wesley, Reading.

Chernoff, H., and Savage, I. R. (1958). Asymptotic normality and efficiency of some nonparametric competitors of the t-test. Ann. Math. Statist. 29, 972-994.

de Finetti, B. (1937). La prévision: ses lois logiques, ses sources sujectives. Ann. de l'Inst. Henri Poincaré ?, 1-68. [Trans. in Kyburg, H., and Smokler, H. (1964), Studies in Subjective Probability, John Wiley, New York.] 
The Neyman-Pearson Theory After Fifty Years

Dempster, A. P. (1983). Purposes and limitations of data Analysis. In Scientific Inference, Data Analysis, and Robustness. Box, Leonard, and Wu, eds. John Wiley, New York.

Diacoris, P., and Freedman, D. (1983). Frequency properties of Bayes rules. In Scientific Inference, Data Analysis, and Robustness. Box, Leonard, and Wu, eds. John Wiley, New York.

Efron, B., and Morris, C. (1973). Stein's estimation rule and its competitors-an empirical Bayes approach. J. Amer. Statist. Assoc. 68, 117-130.

Fisher, R. A. (1922). On the mathematical foundations of theoretical statistics. Phiz. Trans. Roy. Soc. (A) 222, 309-368.

Fisher, R. A. (1935). The Design of Experiments. Oliver and Boyd, Edinburgh.

Freedman, D., and Diaconis, P. (1981). On the histogram as a density estimator: $\mathrm{L}_{2}$ theory. Zeitsch. Wahrsch. 57, 453-476.

Freedman, D., and Diaconis, P. (1983). On inconsistent Bayes estimates in the discrete case. Ann. Statist. 11, 1109-1118.

Hoaglin, D. C., Mosteller, F., and Tukey, J. W. (1983). Understanding Robust and Exploratory Data Analysis. John Wiley, New York.

Hodges, J. L., Jr., and Lehmann, E. L. (1953). The use of previous experience in reaching statistical decisions. Ann. Math. Statist. $23,396-407$.

Hoeffding, W. (1952). The large-sample power of tests based on permutations of observations. Ann. Math. Statist. 23, 169-192.

Huber, P. J. (1981). Robust Statistics. John Wiley, New York.

Jeffreys, H. (1939). The Theory of Probability. Oxford University

Press, Oxford.

Jelihovschi, E. (1984). Estimation of Poisson parameters, subject to constraint. Ph.D. dissertation, University of California, Berkeley.

Lehmann, E. L. (1983). Theory of Point Estimation. John Wiley, New York.

Lindley, D. (1965). Probability and Statistics. Vol. 2: Inference. Cambridge University Press, Cambridge.

Mallows, C. L. (1983). Data description. In Scientific Inference, Data Analysis, and Robustness. Box, Leonard, and Wu, eds. John Wiley, New York.

Mosteller, F., and Tukey, J. W. (1977). Data Analysis and Regression. John Wiley, New York.

Mosteller, F., and Wallace, D. (1964). Inference and Disputed Authorship: The Federalist. Addison-Wesley, Reading. 
Neyman, J. (1937). Outline of a theory of statistical estimation based on the classical theory of probability. Phiz. Trans. Roy. Soc. (A) $236,333-380$.

Neyman, J., and Pearson, E. S. (1933). On the problem of the most efficient tests of statistical hypotheses. Phil. Trans. Roy. Soc. (A) $231,289-337$.

Pearson, E. S. (1966). The Neyman-Pearson story: 1926-1934. In Research Papers in Statistics: Festschrift for J. Neyman. F. N. David, ed. John Wiley, New York.

Raiffa, H., and Schlaifer, R. (1961). Applied Statistical Decision Theory. Harvard University Pres, Cambridge.

Reid, C. (1982). Neyman-From Life. Springer, New York.

Savage, L. J. (1954). Foundations of Statistics. John Wiley, New York. 2nd revised ed., Dover (1972).

Scott, D. W. (1979). On optimal and data-based histograms. Biometrika $66,605-610$.

Stein, C. (1956). Efficient nonparametric testing and estimation. Proc. Third Berkeley Symp. Math. Statist. and Prob. 1, 187-196.

Stone, C. J. (1975). Adaptive maximum likelihood estimators of a location parameter. Ann. Statist. 3, 267-284.

Stone, C. J. (1981). Admissible selection of an accurate and parsimonious normal linear regression model. Ann. Statist. 9, 475485 .

Student [W. S. Gosset] (1908). On the probable error of a mean. Biometrika 6, 1-25.

Tan, W. Y. (1982). Sampling distributions and robustness of $t, F$ and variance-ratio in two samples and ANOVA models with respect to departure from normality. Communications in Statistics, Theory and Methods 11 (No. 22), 2485-2511.

Thisbed, R. (1982). Decision theoretic regression diagnostics. In Statistical Decision Theory and Related Topics. Gupta and Berger, eds. Academic Press, New York. Vol. II, pp. 363-382.

Tukey, J. W., and McLaughlin, D. H. (1963). Less vulnerable confidence and significance procedures for location based on a single sample: Trimming/Winsorization 1. Sankhya 25, 331-352.

Tukey, J. W. (1977). Exploratory Data Analysis. Addison-Wesley, Reading.

Wald, A. (1950). Statistical Decision Eunctions. John Wiley, New York. 